\title{
Baroque
}

\section{La méthodologie des études du baroque}

\section{Francis Courtès}

\section{(2) OpenEdition}

Journals

Édition électronique

URL : http://journals.openedition.org/baroque/512

DOI : 10.4000/baroque. 512

ISSN : 2261-639X

\section{Éditeur :}

Centre de recherches historiques - EHESS, Éditions Cocagne

\section{Édition imprimée}

Date de publication : 1 mai 1980

ISSN : 0067-4222

\section{Référence électronique}

Francis Courtès, "La méthodologie des études du baroque », Baroque [En ligne], 09-10 | 1980, mis en ligne le 15 mai 2013, consulté le 01 mai 2019. URL : http://journals.openedition.org/baroque/512 ; DOI : 10.4000/baroque.512

Ce document a été généré automatiquement le 1 mai 2019.

(c) Tous droits réservés 


\title{
La méthodologie des études du baroque
}

\author{
Francis Courtès
}

\section{Communication}

Les échanges de ce matin, dans leur spontanéité si propre à ne léser personne, paraissent néanmoins avoir été conduits par une idée certaine de la tâche proposée. Aussi convientil d'y revenir en reprendre l'inspiration Je commencerai donc par fixer trois propos dont l'émergence particulière facilite l'orientation, mais dont l'ordre également jalonne un parcours privilégié, qu'il s'agisse d'un fragment de notre itinéraire, ou d'un pressentiment du cercle vicieux où nous sommes menacés de tourner sans espoir.

Le premier, qui a retenti comme un avertissement, est le mot de pessimisme qui a été prononcé dès le début par M. Castan. Il l'a été dans un contexte qui requiert d'être élucidé. Car si notre recherche porte sur l'impensé des recherches sur le baroque, par cela seul elle est critique, et nul ne songera pour elle à la vocation de rassurer. Mais d'autres mots ont été dits, celui de reflux, par exemple, qui renvoient plus précisément à des faits qui ont eu lieu ici. C'est à Montauban, en effet, qu'en 1963 Jean Rousset ${ }^{1}$ a posé cette " question tout à fait folle » : peut-on définir le baroque? Son pessimisme s'en prenait à une définition admise : non, le baroque n'est pas surcharge quand la décoration, même surabondante, sert à révéler la structure. Il souhaitait que faute de couvrir le disparate des références une définition fît état de sa propre difficulté : le baroque devenait alors dans sa réalité comme dans sa notion l'impossibilité de resserrer la synthèse. Le baroque est mouvement d'une tendance à une autre. Mais il suffit d'en vouloir plus pour se voir réduit au silence : la fontaine des Quatre-Fleuves va de l'obélisque au fluent, la chapelle de Saint-Yves des courbes contrariées à la pureté du cercle. Qu'un sonnet joue de ce contraste, ce n'est en toute rigueur que la règle de son jeu, ou le péché de son origine. Mais que la prose de Bossuet ressemble à du Borromini, comme c'est le cas dans tel passage d'une illustre oraison funèbre, voilà dont on doit s'inquiéter : classique, baroque, où est l'antithèse, et dans quel sens la succession? Nous sommes loin des constats simplement négatifs où les critères utilisés pour découvrir péniblement la déviance du baroque pouvaient se contenter d'être mathématiques. Ici ce sont les composantes, les 
catégories nécessaires pour une définition désormais impossible, qui viennent à faire défaut.

Une deuxième remarque, en principe relative au domaine des références, a souligné l'extension prise par la catégorie de baroque dans les faits de la littérature. Malgré les apparences, elle ne contredit pas le pessimisme ou la réserve à l'endroit du concept luimême : on sait bien qu'une voie baroque dans la littérature anglaise a pu être dégagée par un explorateur ${ }^{2}$ qui, n'aimant pas ce mot, ne craignait pas de l'affaiblir. De cette conquête non programmée, Marcel Raymond a rappelé quelques dates ${ }^{3}$ qui excluent le livre d'Eugenio d'Ors, d'une technicité sans doute insuffisante. Mais, tendancieuse ou non, cette chronologie peut nous laisser indifférents. Traducteur de Wölfflin, héritier de ses schémas, Marcel Raymond se préoccupe d'exporter, ou de transposer, un système inventé pour l'œuvre d'art plastique. Il gère prudemment un patrimoine reçu, comme si les données nouvelles n'appelaient pas à restructurer l'entreprise. L'aménagement opportuniste du vocabulaire wölfflinien ignore que le langage, contrairement au dessin, ne pose qu'une touche après l'autre : pictural et non linéaire, c'est par non-résistance, par excès de complaisance, qu'il doit déconcerter le disciple de Wölfflin. C'est le baroque de l'écriture solitaire qui justifie le foisonnement de ces monographies qui nous rendent par fragments, sous la légende des siècles, un discours infini, anonyme, assourdi, tantôt irresponsable et tantôt exigeant, mais critique dans les deux cas. Le langage peut nier parce qu'il miniaturise. Parce qu'il rend dérisoire il juge, condamne, agit. Parce qu'il est sans poids, transportable, il va loin. C'est pourquoi les recherches ponctuelles nous font savoir contre quoi dialoguaient ceux que nous nommons classiques, et de quoi s'entretenait cette voix souterraine dont nous percevons les éclats, mais dont le cheminement, les réseaux, nous échappent. Enfin l'intervention du P. Costabel sur la répartition des hapax chez Descartes comporte des enseignements qui ravivent nos inquiétudes. Elle nous a appris contre les apparences que l'unicité d'une expression tient à ce qui dans l'auteur n'est pas le plus personnel : ainsi quand il varie la forme pour éviter le reproche d'une répétition; ou quand il emprunte une information, à quoi l'on assiste tout au long du livre vII des Météores, dont la langue est exceptionnelle pour l'analyse statistique. Mais à coup sûr l'occurrence la plus intéressante est celle des locutions prises entre deux langages comme des traductions inachevées : ainsi lettre et chiffre, employés de surcroît l'une pour l'autre, rejettent sur la manie de crypter une désignation douteuse ; " égal à rien » est une alliance de mots, concédée à la grossièreté d'un certain bon sens réaliste présumé hors d'état d'en voir l'absurdité. L'hapax devient l'acte manqué, l'abrégé monstrueux d'une conciliation essayée sans plaisir, préjugée impossible; au livre VII des Météores, Descartes fait ce qu'il n'aime pas faire : le pointage de ses lectures ; on comprend qu'il s'applique à ne pas s'en souvenir : l'hapax est le résidu de ce contact déplaisant, qu'il faut laisser derrière soi dans la quête de l'authentique. Si le baroque ${ }^{4}$ est "l'idiome naturel de la culture », dans le droit il précède l'ordre socialisé, dans le fait pour le confondre il le suit et l'imite ; mais si nous dénonçons par là l'inconcevable d'une querelle d'antériorité entre deux attitudes en conflit permanent, nous sommes avertis que l'expression baroque, par sa vocation polémique, peut renvoyer à tous les aspects d'une culture, qu'il s'agisse de ses règles, de ses formes consacrées, ou des contenus intellectuels. Nous risquons d'être amenés à parler de maniérisme par notre propre insuffisance à détecter les allusions et à suivre dans son détail l'explication du singulier. Ou bien quelque chose commence avec l'analyse des langages, ou bien la référence de 
l'œuvre d'art baroque aux constellations culturelles sera l'invincible argument, le dernier mot du pessimisme.

Par la mise en commun de ces observations, elles-mêmes suscitées par des séries de colloques ou intégrées au programme d'une équipe de travail ${ }^{5}$, ce que j'avais à faire a donc été mieux fait qu'il ne l'aurait jamais été par une recherche solitaire. Là est le paradoxe de notre situation. Autour de cette table ronde, sur deux rangs concentriques évoquant un mouvement d'expansion et de contraction, nous avons beau redonner vie au mythe sans image de la circonférence qui n'est plus nulle part alors que son centre est partout, néanmoins nous nous dérobons à l'exigence préparatoire, celle de l'identification; nous travaillons en groupe sur un objet qui ne paraît pas comporter l'existence en groupe : nulle part il n'est question d'une école baroque, on ne se sert pas de ce mot pour désigner des collections mais à la rigueur des personnages, principalement des œuvres ou des projets. Tout se passe comme si le mimétisme initiatique ou initial avait perdu sa raison d'être, et comme si, chez les tard-venus que nous sommes, les études du baroque d'elles-mêmes et sans nous, cherchaient en nous leur second souffle. Elles ont à sortir de l'ère wölfflinienne pour faire face à un afflux illimité de documents. Elles manquent d'on ne sait quoi, sans doute d'unité dans la motivation. De leur quête nous sommes avant tout les témoins.

5 La même explication nous sera suggérée par un deuxième paradoxe qui, développé de toutes les façons que l'on voudra, nous décourage de formuler la question méthodologique.

61 - En 1921, Eugenio d'Ors imaginait, à l'entrée de la forêt du baroque, les deux colonnes ${ }^{6}$ du prophétisme, l'une d'elles étant l'Apocalypse. «Voyez, je fais toutes choses nouvelles »: voilà ce qui est promis par la révélation ${ }^{7}$. Or, il est évident que toute monographie s'annonce comme une révélation: ce qu'elle vient ajouter à la nomenclature des modèles déjà reconnus pour baroques lui semble à même de transformer la connaissance du baroque, de marquer le privilège d'un baroque défini, de fournir l'aiguille aimantée, le principe hiérarchique désignant tel baroque pour éminent ou originaire. À suivre cet exemple, nous pourrions présenter une communication sur un modèle supplémentaire, ou bien un rapport, un bilan, une philosophie positive, une coordination des différents modèles dans « l'ensemble des moyens propres à dévoiler ». Mais l'attente de ces journées n'a été comprise par personne comme celle d'un recensement.

72 - Ce que fait espérer un effort d'attention méthodologique aux études sur le baroque, n'étant pas la surcharge d'une révélation, devra s'appeler prise de conscience. Précisément ce mot, bien loin de proposer une libération envers les préjugés d'une étude sectorielle, en comporte certains qu'il convient de relever. La conscience, comme la souffrance qui en est l'éveil ordinaire, est toujours celle de quelqu'un. Elle requiert un porteur, humain en l'occurrence. Il nous reste une preuve à fournir, que les études du baroque intéressent l'homme avant le chercheur, que la distance entre elles et l'homme n'est qu'apparente et artificielle, entretenue par un rituel objectiviste, complaisamment cérémonieux. Si c'est de nous qu'il s'agit, me voici mis en demeure d'amener jusqu'à chacun de nous, en tant qu'homme simplement, sans détermination de compétence 
scientifique, la problématique du baroque. Dès lors, malheureusement, pour prévenir le danger qui pèse sur l'empirisme (celui de sortir de la question), on sera fixé à soi-même ; c'est hors de nous, à nos dépens, que sera rempli notre programme par l'observateur étranger qui affirmera savoir d'avance le peu que nous pourrons lui dire. Il est vrai que nous pourrons faire valoir ce jeu sous la forme (hélas prévisible) d'une communication à caractère éthique : introduire la redondance dans le moule canonique de l'exposé factuel, c'est une manière de performance.

3 - Le baroque comme mode d'existence de l'homme simplement tel aurait néanmoins l'avantage de pouvoir être réfléchi, c'est-à-dire égal à lui-même de part et d'autre du regard. Ce n'est pas le cas, on le sent bien, pour le baroque comme discipline et choix esthétique. Et pourtant on hésite à faire table rase de tout contenu. Les moralistes rigoureux qui ont fondé en France l'école sociologique ne répugnaient pas aux monographies, bien que la matière de celles-ci ne fût pas toujours édifiante. Libre à nous de penser, à leur imitation, que dans une étude baroquisante la vérité sur le baroque est présente, mais "quelque part ", sous une forme différente de la rédaction adoptée. Mais le penser vraiment c'est faire qu'elle advienne. Le programme d'une étude, posé comme extérieur (éventuellement comme insolite) cherche dans la culture de l'auteur et des lecteurs ses termes de comparaison: il se prolonge vers des limites qui ne sont pas définitives puisqu'elles portent son reflet avant de le lui rendre. La réflexion se passe de subjectivité.

9 Le dialogue qui lie l'auteur à son lecteur n'engage pas des personnes, mais des degrés de rigueur. Montrons-le par l'absurde. Si je m'identifie à l'auteur que je lis, sa communication est vécue sur la base d'une définition du baroque, à laquelle se conforme le texte qu'il a écrit. Mais ma propre lecture a dû faire l'essai de Quelques autres avant de s'insérer dans la bonne. Moins exigeant par crainte de l'être à contresens ou en dehors d'une exigence que j'ai tout d'abord mal perçue, maladroitement docile, encore peu responsable, j'ai recréé, par mes timides aventures autour d'un concept présumé, une constellation idéale. La connivence mal éclairée dispose aux parallèles et aux coordinations. Ce qu'est le fait total pour le sociologue est pour la distraction féconde du lecteur la multiplicité des significations, segmentée en concepts au profit du discours et de son déroulement. Le chercheur n'a part à l'idée qu'avant de préciser son thème ou de formuler sa découverte. Les promesses qu'on lui impute sont honorées différemment.

Encore est-ce avec joie qu'on se résignerait à la consolation que serait le concept. Mais le scrupule de ceux qui avouent ne pas savoir si leur monographie s'inscrit dans' le baroque, comme l'absence de scrupule de ceux qui estiment qu' " on ne sait jamais ", accuse un autre déficit. Loin de moi la pensée de condamner ceux-ci : rien n'interdit que le savoir soit un jour débiteur de leur agnosticisme. L'absence du concept ne provoque pas seulement des conduites de mauvaise foi, telles que diversion ou fausse modestie. L'intérêt de la recherche peut y trouver son compte : aussi a-t-elle été, une fois au moins, souhaitée, dans un éditorial que je citerai tout à l'heure. On ne voit pas assez qu'elle redevient consciente avant tout accroissement des études du baroque. Est-ce assez pour conclure Qu'elle les détermine, ou qu'elle est partie intégrante de l'interrogation qui les fait progresser? Du moins suis-je cette fois justifié dans l'usage que je ferai encore de ces constats d'échec. 
Il faut citer en son entier le passage de Marcel Raymond, qui paraissait aller de soi quand les études sur le baroque, en langue française, étaient modestes, mais qui mérite désormais une attention plus appuyée. Il fait partie de la conclusion de sa première conférence sur le baroquisme littéraire, telle qu'elle a été publiée en 1948 dans la collection des Cahiers du Collège philosophique ${ }^{8}$. Il nous dissuade «de faire entrer les écrivains de cet âge qui va de Ronsard à Racine dans un système fermé de pensée, dans un système esthétique ». Première remarque: sachons que l'emploi du mot système, dans le sens de concept appliqué, comme synonyme de lieu clos, ne représente au sein de la langue philosophique qu'une convention particulière, laquelle ne serait pas admise notamment par un lecteur d'Auguste Comte soucieux de s'élever à la philosophie (comme système général des connaissances humaines) et non de professer une philosophie; l'étude des éléments peut être dominée " essentiellement... par celle du système » sans que la théorie veuille se donner pour plus qu'une coordination des faits que l'on observe, à condition que l'observateur ait une deuxième ambition, contemporaine de son travail. Ce serait une étude d'un très haut intérêt que de se demander dans quelle perspective le mot système est restrictif, et s'il ne l'est pas par hasard sous certaines influences (psychologistes, subjectivistes) auxquelles Comte s'est opposé.

Voici la suite : "On se tromperait en dessinant un pays du baroque, dans la littérature française, qui aurait des frontières précises et dont tous les ressortissants seraient porteurs du même passeport. L'essentiel est de se mettre en mesure de voir mieux et de mieux comprendre avec leurs caractères propres, des œuvres que l'on a trop souvent négligées, ou jugées sommairement, par rapport à une échelle de valeurs qui ne leur convient pas. Il importerait de les rattacher à leurs temps, puis de les éclairer latéralement, en examinant par où elles ressemblent aux productions contemporaines des beaux-arts ».

On pourrait distinguer trois niveaux dans ce texte :

1 - Celui de la timidité, ou si l'on veut de la bienséance. L'auteur s'excuse et prie en somme que l'on n'attende pas trop de lui;

2 - Mais, au lendemain d'une guerre où il avait fallu savoir perdre pour la gagner, la modestie contaminée par la philosophie de l'histoire prend facilement les dimensions d'une morale de la faiblesse; d'où l'amplification politique immergée sous la métaphore du passeport, et la traduction vraisemblable : n'attendez pas de nous des classifications, des cloisonnements qui feraient penser aux baraques et aux clôtures d'un camp de prisonniers, car nous avons le respect de l'homme (en ce temps-là on lisait avec attendrissement la Lettre à un otage de Saint-Exupéry). Que contient cette deuxième couche, outre cette promesse humaniste (en d'autres termes: négative)? Voyons la phrase finale de cette conférence : «Pour les créateurs authentiques, les fatalités d'époque ${ }^{9}$ perdent leur force contraignante ». Acceptons de comprendre à travers ce propos que l'exemption de fatalité n'est pas au crédit du baroque, le créateur soustrait aux prises du destin n'étant pas baroque, mais classique. L'œuvre baroque a une date, qui est celle de l'antagonisme; faute d'intégration ou de dépassement elle reste inférieure par son indiscipline. Impossible d'être plus dur pour l'historien Marcel Raymond: le critique qu'il est, censeur de l'inachevé, du passionnel, des sous-produits ${ }^{10}$, ne saurait approuver la piété ou le zèle égarés qui les ressuscitent. Ou bien le meilleur humanisme est celui qui efface l'homme. Dans la représentation sadique et masochiste (ou si l'on aime mieux: 
valéryenne et gidienne) qui fait du classicisme un baroque surmonté, si l'écriture met un terme à l'instabilité de l'ordre, c'est pour une conclusion que l'on n'aurait pas prévue ;

3 - Essayons notre chance au troisième niveau, celui qui devrait être épistémologique : Marcel Raymond demande la permission de juger sans identifier par le moyen du prédicat. Observons que ce n'est pas d'étiquette qu'il parle : les étiquettes servent à reconnaître, en vue d'une certaine pratique. Le choix du passeport comme symbole évidemment péjoratif proteste contre une décision de caractère théorique : le passeport étant une pièce d'identité , marquant la limite supérieure de la curiosité de la police des frontières, et pouvant même servir de gage à l'autorité qui le retient, le prédicat, une fois admis que le sujet lui est identique, totalise le savoir possible ou désirable à son égard, et l'enferme si bien que le mépris s'en empare : la souveraineté du prédicat sur les êtres qui ne sont que lui les fait strictement remplaçables.

Les amateurs prennent volontiers la méfiance qu'inspire à bon droit leur idée du rationalisme pour une critique fondamentale du rationalisme lui-même. Le contact de Marcel Raymond avec les problèmes du connaître lui vient sans doute uniquement de ce qu'il avait lu pour écrire en 1946 un essai ${ }^{11}$ sur Paul Valéry. C'est dans la Note et digression qu'il a relevé, pour la retenir, la possibilité que la substitution soit le caractère des choses comme telles. Dans le Bilan de l'intelligence qu'il a noté au sein de l'homme la division entre son être et son savoir, irréparable, le machinal prenant le pas sur la qualité. Aussi n'y a-til pas lieu d'expliciter l'accord entre vœu de lucidité et subordination requise de l'esprit. L'exégèse ne consiste ici qu'à faire crédit de tout ce qui n'est pas formellement exclu.

On sait bien que Marcel Raymond n'avait pas lu la part diurne et laborieuse des ouvrages de Bachelard. C'est le respect des raisons qui réclame l'Essai sur la connaissance approchée ${ }^{12}$. Le concept y est ce qui provient de la conceptualisation, laquelle en tant que travail a vocation de progrès. Or ce qui précède le prédicat n'est pas l'antéprédicatif: «la psychologie moderne a, comme on le sait, surabondamment établi l'antériorité du jugement sur le concept ». Moment, relais, agent d'une transformation, le concept n'est « représenté » que par une sorte de violence faite à l'élan d'une genèse ; tel un instantané sur une oscillation, il se trouve alors " enveloppé d'une atmosphère qui en rend le contour imprécis». Au nom de quoi décréter une limitation et "un arrêt dans l'analyse »? On ne pense pas avec des concepts séparés, la pensée n'est contemporaine que «de la jonction des concepts». Bref, à l'état naissant, au stade créateur, la connaissance est faite de jugements synthétiques dont l'effort est soit d'annexer une théorie déjà construite, soit de poursuivre une exploration. Le destin du concept est d'être rectifié sur une expérience nouvelle, et « en changeant d'ambiance le concept change d'allure ». De même que l'arithmétique enrichit de son propre bien toutes les opérations qui lui sont empruntées, le changement du sujet porteur retentit sur le prédicat. La menace d'aliénation du sujet par le prédicat est remplacée par son contraire : «la compréhension est une fonction très mal déterminée de l'extension ». Priorité à l'extension, pourvu qu'elle soit articulée : « une connaissance poussée par des méthodes d'approximation pourra suivre le phénomène jusque dans son individualité et son mouvement propre ». Où s'organisent les concepts leurs connexions sont à l'écoute des vibrations originales. La crainte qu'on a d'un concept irait plus justement à son unicité, au contresens qui le sépare de sa dialectique irréversible. 


\section{l'antagonisme peut correspondre à des limites. Faute du pouvoir de variation, dont} l'historien est dépourvu, on peut concevoir sans trop de peine un excès, un caprice qui consisterait à dire: un discours sans concept, nous relevons le défi ; parlons d'abord, selon le besoin et l'occurrence, suivant la direction, la connexion des choses. Plus tard, le temps viendra de nous justifier devant la police logicienne, de présenter le fameux passeport, d'attester que nous sommes restés dans le baroque. L'autre attitude serait le dépit: faute de fixer la règle on arrête le jeu, une variante consistant à forcer le changement de règles, par exemple en parodiant les désignations linnéennes à la façon d'Eugenio d'Ors ${ }^{13}$, jusqu'à se demander si le baroque existe. Certes le vrai problème est celui d'inventer, dans l'intervalle de ces limites, les règles propres à entretenir l'animation de la partie. Mais, qu'il y ait une vérité dans l'outrance et dans l'humour noir, c'est ce qui va ressortir d'un exemple a contrario décidément privilégié.

Il consiste dans l'exposé présenté par Pierre Mesnard ${ }^{14}$ aux journées de 1963. Loin de nous le projet de discuter ici la désignation de Leibniz comme philosophe du baroque, cette improvisation sans doute publicitaire ne méritant pas de survivre. Insistons sur l'itinéraire, la prétendue méthode dont elle se réclame. Essentiellement son postulat est que le philosophe arrive toujours après : il faut donc percevoir le tout de la période pour situer dans le temps son siège présumé. L'indice chronologique devrait être, en principe, le sens de tel moment, individualisé, d'après sa place le long de ce tout: balbutiements. croissance, plénitude, vieillesse. Qui ne voit dès lors qu'en deux langages (expression de la phase dans l'auteur, ou bien contribution de l'auteur à la phase) on décrit les mêmes données ? Mais que l'équivalence ne soit pas prise comme telle, l'histoire étant considérée comme discipline par concepts, et l'on ira tout droit de la tautologie à la fausse détermination.

C'est bien ce qui se produit dans l'exposé de Mesnard. De la priorité reconnue au concept et, sans critique, transférée à l'outil de remplacement qu'est l'indice chronologique, la conclusion est que le cycle culturel détermine le sens des faits qui le composent. Pas de baroque en dehors de certaines frontières. Précautions renforcées pour les périodes voisines. Qu'il s'agisse du baroque ou de la Renaissance, entre forme et période existe « la même loi de l'implication réciproque ». D'où l'étrange contradiction de la fin poursuivie et des résultats obtenus: Mesnard s'est proposé de sauver Raphaël de l'accusation de maniérisme. Son but est donc ouvertement la connaissance de l'individuel; mais parce que pour lui connaître exige « identifier, analyser et classer les hommes, les œuvres et les époques ", cette défense n'aura lieu que par étouffement de la notion de maniérisme (inadmise comme fait de civilisation) et sous la condition que Raphaël réintègre une catégorie plus noble : il sera, de la Renaissance, l'un des «maîtres les plus accomplis ». Classe, classement, affaire classée. La recherche du type qui nous est recommandée n'est pas celle du type au sens exclamatif, faite pour exalter l'émergence et le différent, mais pour symboliser l'allégement de la mémoire, l'abréviation par la mémoire, bref l'économie de pensée.

19 Aussi ferons-nous un sort au cliché qu'il répète, celui de la contrariété de la compréhension et de l'extension. Qu'elles varient en sens inverse dans le maniement d'un concept, cela dépend d'une hypothèse qui est la fixité des moyens conceptuels. Car si le budget de l'intelligence n'est pas stabilisé par décret de la paresse, si l'élucidation 
progresse effectivement, l'accroissement de la richesse n'exige plus la compensation, n'impose plus l'affaiblissement comme prix de la dispersion, et ne craint pas l'échec devant le singulier. Les sciences humaines appellent désormais une forme d'attention à l'enchevêtrement qui ne soit plus en danger de repli dogmatique sur le balisage extérieur. L'analyse n'est qu'un mot si l'on ne fait qu'enregistrer, au lieu de vouloir, la différence. Parfois le savant sait plus avec moins de données, reconstituant le divers sinon le successif dans le signal du phénomène. Ce n'est plus la matière à traiter qui est tenue de faire le poids.

Ce rêve d'un outillage productif et léger, le quatrième Cahier ${ }^{15}$ de cette revue en a fait l'aveu en toutes lettres. «Plus la notion de baroque se précise comme pur néant, plus il y a chance qu'elle serve efficacement l'analyse de civilisation, instrument sans épaisseur et sans opacité de la méthode scientifique ". C'est dans le même esprit qu'à la phrase suivante il est question d'une eau-miroir, sans tain et sans rigidité, forme extrême de non-résistance au regard, de non déformation de la chose regardée, pourvu naturellement qu'elle y soit tout entière. Dans cette évacuation de ce qui pèse et qui trouble; mais aussi de ce qui arrête, il faut donc lire le symbole d'une recherche non restrictive. Si l'on ne sait pas ce qu'on cherche, on ne saura pas ce qu'on trouve : c'est la logique volontariste. Mais si l'on sait ce qu'on cherche, saura-t-on de surcroît accueillir le déconcertant, et tout simplement l'ignoré ? Le «lâchez tout » surréaliste, avant de " partir sur les routes » veut qu'on perde réellement « pour laisser place à la trouvaille». parrainage des grands maîtres de ce calcul différentiel qui s'appelaient Maupertuis, Euler et d'Alembert, dont le rationalisme au temps de Frédéric II ne venait pas à bout des survivances baroques à l'Académie de Berlin. Chez les mystiques par contre le thème du savoir qui me prive de tout ce que je pourrais apprendre a connu bien des traductions qui seraient ici mieux à leur place. C'est, dit Silesius, faute de vide intérieur, de réceptivité, que nous ne pouvons contenir Dieu : il vit de la mort de notre moi ${ }^{16}$; nous serons libres pour lui quand nous ne serons rien : « laissez le quelque chose ». Nous n'avons pas fini de noter l'isomorphisme entre les faits baroques et l'approche qu'ils réclament: par égard pour la viduité requise pour la venue de Dieu, n'en sachez pas trop en entrant; vous risqueriez à la sortie de ne pas en savoir davantage.

Posant cette pierre d'attente j'aimerais en relever deux que j'ai laissées un peu plus haut. L'une concerne les œuvres que selon Marcel Raymond « l'on a trop souvent négligées » ou mal jugées au nom de valeurs qui ne leur étaient pas destinées, l'autre se rapportant aux «drames collectifs », aux divisions de l'histoire qui selon Pierre Mesnard comprennent le baroque entre deux civilisations, la Renaissance et le classicisme. Ici «l'âge baroque » serait lié sans doute à une certaine culture, tributaire de la prépondérance espagnole, pendant l'absence d'une culture tenue, elle, pour irrécusable ; là l'absence est pour ainsi dire dans le regard de l'historien. Négation objective : l'authentique fait défaut; négation subjective : refus d'authentifier. Encore l'isomorphisme.

Conserver ce qui fut nié, racheter la part maudite : vocation du baroquisant. Mais avant d'en venir là nous avons à penser l'idée même de négation. Qu'est-ce, pour le logicien, 
sinon l'altérité ? Le rappel nécessaire de la comparaison dont elle est le procès-verbal nous protège à peine du quelconque. À preuve, les chapitres où il faut chez Lanson se mettre en quête des baroques : l'un se rapporte à ce qu'ils précèdent et n'est circonscrit que par là. L'autre curieusement s'intitule Attardés et égarés : attardé, en principe, on ne l'est que par rapport à la vitesse de l'histoire, et à cause du poids du passé ; c'est ainsi que l'on peut au dix-septième siècle être un combattant du seizième: un tel jugement (sommaire, porté sur d'Aubigné) n'a de termes que positifs. Égaré, pareillement, on l'est par adhésion, dans son propre pays, à des goûts étrangers : italiens, espagnols. Mais que dire d'un Scudéry quand il donne à ses Wisigoths des traits qui ne sont d'aucun temps? Qu'il manque de génie ? C'est bien ce que fait Lanson. Mais l'on ne peut spécifier ni cette sorte de manque, ni ailleurs le rapport du burlesque au précieux. Il y a mille façons de n'être ni génial, ni convenu, ni naturel.

Les études du baroque nous semblent commandées, dans ce qu'elles ont de conceptuel, par cette nécessité de quadriller le quelconque, poussé jusqu'à l'imprévisible. Nous soulignerons donc dans l'œuvre de Wölfflin le développement implacable de cette situation épistémologique : ce qui s'exprime en lui, qui vient au jour par lui, est la souffrance du concept. Premièrement il a écrit pour le baroque (c'est-à-dire : au nom du baroque) cette littérature qu'il n'avait pas connue. D'où la fameuse série de couples de concepts formant la table des matières de ses Principes d'histoire de l'art. Il n'a pas eu la chance qu'aura Eugenio d'Ors de tomber amoureux d'une catégorie: même si c'est une femme fatale, celle que l'on poursuit de Vienne au Portugal, pour qui les palmiers de Coïmbra caressent l'air de leur éventail ${ }^{17}$, ce n'était pas un tourbillon; elle ne harcelait pas comme les Euménides. Faire cinq fois l'expérience de mener une double vie, pour épuiser comme Don Juan les figures de l'altérité, cela par contre n'est plus une vie, mais bien une combinatoire. Wölfflin a écrit les Principes comme Freud avait déchiffré le grand rêve $^{18}$ de la Traumdeutung, au risque des pires surprises. Où est l'épreuve immédiate qui l'assure d'avoir gardé le même cap ? L'obscur et le profond, quand il s'agit de peinture au lieu de philosophie, ne requièrent-ils pas la médiation de l'art pour devenir conciliables ? L'unité et la forme ouverte ne se contredisent-elles pas ? Il importe à l'intelligence, quelle que soit l'opinion consciente de Wölfflin, qu'il y ait diversité, même s'il y a dérapage, comme il importe qu'il y ait couple, afin qu'il n'y ait pas d'espace non parcouru, mais qu'il $\mathrm{y}$ ait toujours de quoi nommer l'apparence particulière. Ce pluralisme non cohérent remémore la circonstance, la situation fondamentale qui a initialement réclamé le mot baroque, sans lequel rien n'aurait permis de qualifier la littérature atypique et parfois rugueuse d'un pays comme l'Allemagne, déchiré au temps de la Renaissance accablé depuis l'année de la Melencolia, et privé de classicisme jusqu'au temps des lumières.

Deuxièmement, selon un ordre qui n'a rien de chronologique, la prolifération des critères garde le sens de la négation qu'elle recouvre. Nous inférons de leur discordance qu'ils ne seront pas toujours réunis, leur pouvoir de contestation les opposant les uns aux autres. L'historien qui feint la surprise, et se réjouit en secret, de la non-coïncidence entre une appellation (évidemment conventionnelle) et une définition (ici sursaturée) ne sait pas qu'il travaille au service de l'idée. S'il objecte un baroque, éventuellement voulu (plein ou artificiel, au gré des sollicitations) en dehors du cadre historique, souhaitons-lui le talent de nous faire oublier que la valeur polémique de cet anachronisme tient au dogme naif d'une superposition entre la forme et la période : la loi de Mesnard contre Wölfflin, ce serait du meilleur burlesque. Rien n'empêche pourtant que cela soit prévu par l'autodestruction nécessaire du concept. Aux yeux du logicien, sitôt déterminée la forme est en 
quête d'emploi, universellement disponible, pour tout sujet à quoi elle pourrait s'appliquer : là où elle le peut à l'exclusion des autres elle réfute le lien qui est censé les unir. Dès lors le chemin fait par Wölfflin de Renaissance et baroque aux Principes devient celui du sacrifice. Dans la théorie ébauchée en 1888 les critères mis en avant s'étagent sur plusieurs registres; ils peuvent être compris comme simples descriptions d'une réalité visible, comme évocations imagées d'une présence dynamique, ou bien d'une sensation rapportée au corps propre : spectacle, masse, ou mouvement. De cette polysémie pouvait toujours venir une possibilité de conciliation. Or, les Principes commencent par creuser l'antithèse du linéaire et du pictural. Qu'y a-t-il là qui soit de nature à réveiller le Sens du vécu, chez un disciple de Dilthey, ou, chez un fidèle de Volkelt, la conscience projective? L'art n'a d'histoire que la sienne et c'est principalement l'histoire de la vision. Désormais, plus d'échappatoire du côté de l'invérifiable. Il va falloir cette fois que le destin s'accomplisse, et qu'on expie la faute de conceptualiser ${ }^{19}$. traité du baroque, à défaut de convaincre, se fera reconnaitre au sentiment qu'il donne d'être parent de son contenu. C'est ce qui d'entrée de jeu frappe chez Eugenio d'Ors : «ce livre est un roman autobiographique». Sait-on ce qu'est un roman? «Narration ${ }^{21}$ vraie ou feinte, écrite en vieux langage ou langage roman». Le baroque fait des tentatives dans les marges de la culture ; l'office du roman est de fournir une culture à ceux qui n'ont pas le langage-véhicule de la « vraie » culture : l'homme aux quarante écus est ainsi le roman de l'économie politique; non pas la parodie (qui copierait sa langue), non pas la vulgarisation (silencieuse sur les drames). Il fallait que l'ouvrage de d'Ors fût un roman, parce que s'il y a jamais eu une préhistoire du baroque, le roman doit y figurer : l'affinité est évidente.

Autobiographie ? Les Principes de Wölfflin disent la vie d'un homme transformé en esprit Mais Renaissance et baroque parlaient d'une jeunesse dévorée par l'émulation. Aussi l'émulation en sera-t-elle l'objet. Au centre est Michel-Ange inquiet de son génie, n'ayant d'autre salut que la majoration. En plein cœur, la morsure d'une phrase ${ }^{22}$ de Burckhardt : « l'art baroque parle la même langue que la Renaissance, mais il n'en parle qu'un dialecte corrompu ». Pari grandiose : je ferai mieux que lui, et l'on respectera le baroque. Qui est réellement le rédempteur de qui ? Wölffiin sera brillant, savant et pénétrant: comme 
Le Bernin, comme Vignola, plus que Burckhardt ; et l'avenir dira : comme Wölfflin. Il n'est pas de baroquisant qui ne soit, en un sens, baroque.

\section{NOTES}

1. Actes des Journées internationales de Montauban de 1963, pp. 19 à 23.

2. Jean-Jacques DENONAIN, "Aspects du baroque littéraire en Angleterre ", ibidem. p. 73 à 84 .

3. Baroquisme et littérature, pp. 157 et 158 . Voir ci-dessous, note 8.

4. Le mot que nous citons est d'Eugenio d'Ors, in Du baroque, p. 132.

5. Présentée par P. costabel et J.-R. ARMogathe : Primo colloquio internazionale del lessico intellettual europeo, 1974, pp. 113 à 121.

6. Du baroque, p. 38.

7. Apocalypse 21,5

8. Sous le titre collectif La Profondeur et le rythme. Le passage que nous citons est aux pp. 164-165.

9. Ibidem, p. 167.

10. Textuel, p. 166.

11. Paul Valéry et la tentation de l'esprit, à La Baconnière, Neuchâtel, 1946. Les emprunts que nous lui faisons proviennent de son dernier chapitre, La civilisation et la crise de l'esprit.

12. Paris, Librairie Vrin, 1927. Nos citations renvoient au deuxième chapitre, La recti fication des concepts.

13. Du baroque, pp. 155 à 173.

14. Recueil cité, pp. 37 à 42.

15. Éditorial, p. 3.

16. Le Pèlerin chérubinique, I, 44, 128 ; V, 126, 205.

17. Du baroque, p. 36.

18. La Science des rêves, chap. II.

19. Cf. Bernard TEYSSÈDRE, Présentation de Renaissance et baroque, p. 26.

20. Ennéade V, traité VI, § 5.

21. Dictionnaire... de Littré.

22. - Dans le Cicérone (1854) ; citée par Marcel Raymond, p. 141.

\section{AUTEUR}

\section{FRANCIS COURTÈS}

Université de Montpellier 